\title{
Low Complexity Scheduling Algorithm for the Downlink of Distributed Antenna Systems
}

\author{
Eduardo Castañeda, Ramiro Samano-Robles, and Atílio Gameiro, \\ Instituto de Telecomunicações, Campus Universitário, Aveiro, 3810-193, Portugal. \\ \{ecastaneda,ramiro\}@av.it.pt, amg@ua.pt
}

\begin{abstract}
In this paper we present a low-complexity user selection algorithm for the downlink of a distributed antenna system (DAS) that achieves an optimum solution for a weighted matching problem. The user selection process is modeled as a linear sum assignment problem (LSAP). The proposed solution consists of two phases. In the first phase, a set of potential users to be scheduled is found by combining two complementary approaches: greedy and minimum-throughput-loss selection. In the second phase, the set of scheduled users is refined by selecting the users that maximize sum throughput. We provide numerical results to confirm the optimality of our user selection algorithm and to compare its performance with existing solutions.
\end{abstract}

\section{INTRODUCTION}

The achievable data rate in wireless communication system is limited by the available number of radio resources. As the number of simultaneous users and services increases, the efficient design of the resource management policies and the air interfaces in wireless networks become fundamental to meet the users requirements without increasing the system bandwidth or power budget. A solution to this problem can be found in the area of distributed antenna systems (DAS). These type of air interfaces are based on the concept of space diversity and cell splitting in order to achieve high spectral efficiency. In DAS architecture, the remote antenna units (RAUs) are geographically separated and connected by a dedicated link to a central unit (CU) where signal processing is jointly performed. The idea behind DAS is to reduce the access distance to the user, increasing its signal-to-interferenceplus-noise ratio (SINR). DAS have been proved to increase the coverage, reduce the inter-cell interference in outdoor applications, and reduce the power consumption [1]. Since the $\mathrm{CU}$ processes all the signals of the RAUs, cooperative and collaborative algorithms for resource assignment can be implemented.

In multi-user multiple-input multiple-output (MIMO) systems where the antennas at the transmitter are co-located, the most common criterion for selecting users for downlink transmission is based on the orthogonality or correlation between wireless channels. In [2] the authors propose a user selection based on graph theory where the criterion to group users served by the same spreading code in a MIMO-CDMA system is defined by their nearly orthogonal spatial signatures. Using graph coloring techniques, users that can create interference

The work presented in this paper was supported by the Portuguese FCT CADWIN (PTDC/EEA TEL/099241/2008) and CROWN (PTDC/EEATEL/115828/2009) projects. between each other are isolated and users with relative low values of interference are served in the same radio resource. A low-complexity algorithm for user selection was proposed in [3] where the set of selected users minimizes the sum of the channel correlation. As the minimum average correlation metric is not enough to guarantee the largest sum rate, an improved version of [3] was proposed by Karachontzitis [4] where the scheduling decisions are made evaluating iteratively the correlation and the achievable sum throughput of each possible set of users.

Although extensive work has been done for user selection in MIMO systems combining metrics such as spatial correlation and achievable throughput, such selection schemes cannot be applied directly to distributed antenna systems as we have to account for different path losses. In the case of DAS although some work has been published, the topics relative to user selection still need research to achieve maturity. The scheduling strategy to maximize the system throughput is based on selecting the user with best channel conditions. This is the well known channel-aware scheduling, which exploits instantaneous channel conditions and multi-user diversity. Authors in [5] implement a channel-aware scheduling by proposing a user selection algorithm based on the statistical characteristics of the instantaneous large-scale fading for DAS. A greedy algorithm finds the set of dominant users for which the difference between their instantaneous channels lies below a threshold.

In this paper a cooperative approach between RAUs is proposed to define the set of users for which their sum throughput is the largest. This kind of cooperation resembles a particular class of matching problem known as linear sum assignment problem (LSAP) [6]. We define a methodology for user selection to be used in the downlink transmission for DAS, that finds an asymptotically optimal solution of the LSAP by using a low-complexity matching algorithm. Our methodology can be described in two phases. In the first phase, two complementary algorithms define together a set of potential users for scheduling with the best individual achievable throughput combining two different philosophies: a greedy user selection and a minimum-throughput-loss user selection. In the second phase a third algorithm evaluates from the set of potential users previously found, the final subset of scheduled users that maximize the total sum throughput.

The remainder of the paper is organized as follows. In Section II the system model used for the DAS scenario is 
defined. Section III exemplifies the user selection problem addressed in this paper. Section IV describes the proposed algorithms for user selection and throughput maximization. Section V shows the performance evaluation and numerical results. Finally conclusions are drawn in Section VI.

\section{SySTEM MODEL}

We consider a single cell wireless communication system with $K$ active users and one CU equipped with $M$ RAUs.The user terminals and RAUs are single antenna. The channel between user $k$ and the RAU $m$ is block fading and modeled as a zero mean circular symmetric Complex Gaussian random variable, $h_{k, m} \sim \mathcal{C N}\left(0, \sigma^{2}\right)$. The channels are affected by a path-loss component $L_{k, m}$, and a shadowing fading component $\psi_{k, m}$ modeled as a log-normal distributed variable with parameter $\sigma_{s}$. The signal received by the user $k$ is defined as:

$$
y_{k}=\mathbf{h}_{k} \mathbf{x}+z_{k}, \quad \forall k \in\{1, \ldots, K\}
$$

where $\mathbf{x}=\left[x_{1}, \ldots, x_{M}\right]^{T}$ is the transmitted symbol vector by the RAUs, $\mathbf{h}_{k} \in \mathbb{C}^{1 \times M}$ is the channel vector to the $k$ th user, $z_{k}$ is the additive white Gaussian noise with variance $\sigma_{z}^{2}$ at the $k$ th user. It is assumed that $\mathbb{E}\left[x_{i} x_{i}^{*}\right]=p, \forall i$ and that we have perfect CSI at the CU. Considering that all RAUs have allocated power $p$, the SINR $\gamma_{k, m}$ experienced by the user $k$ th in the $m$ th RAU can be expressed mathematically as:

$$
\gamma_{k, m}=\frac{p\left\|h_{k, m}\right\|^{2}}{\sum_{n=1, n \neq m}^{M} p\left\|h_{k, n}\right\|^{2}+\sigma_{z}^{2}}
$$

Each user feeds back its channel gain to the $\mathrm{CU}$ where the scheduling processing is performed. The achievable throughput of the $k$ th user selected to be scheduled in the $m$ th RAU is given by:

$$
R_{k, m}=\log \left(1+\gamma_{k, m}\right)
$$

\section{User Selection Problem}

Let us consider a cellular system with $K$ active users and one CU equipped with $M$ RAUs. The CU must define a set of users that maximizes a specific global parameter such as throughput, fairness, QoS, etc. The optimum set of users in this scenario can be found within a search space of size ${ }^{1}{ }^{K} \mathbf{P}_{M}+$ $M\left(\sum_{m=1}^{M-1} K \mathbf{P}_{m}\right)$, for $K \geq M$ which is prohibitively large for high values of $K$. In order to reduce the complexity of the user selection, the algorithms proposed in this paper analyzed the search space of size ${ }^{K} \mathbf{P}_{M}$ where all RAUs transmit at the same time.

In order to illustrate the users selection in this search space, consider the system in Fig. 1 for a single radio resource, constraining all $K$ users in $V_{2}=\{1,2,3\}$ to have the same priority, all $M$ RAUs in $V_{1}=\{a, b, c\}$ to schedule only one user, and it is assumed that there is always information to be sent to the users (full buffer). The links between the users and the RAUs represent the achievable throughput given by (3) and

\footnotetext{
${ }^{1}$ The search space are all possible combinations that $M$ RAUs can schedule $K$ users having at least one RAU transmitting. ${ }^{K} \mathbf{C}_{m}$ and ${ }^{K} \mathbf{P}_{m}$ denote respectively, the combination and permutation numbers of $K$ elements in $m$ places.
}

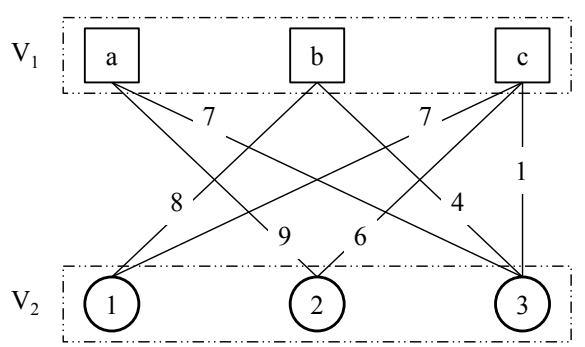

Fig. 1: A wireless network with $M=3$ RAUs $\{a, b, c\}$ and $K=3$ users $\{1,2,3\} . V_{1}$ is the set of all RAUs in the system and $V_{2}$ is the set of active users in the system.

can be expressed in the matrix $C \in \mathbb{R}^{K \times M}$. One approach to generate the set of scheduled users is to find iteratively the link with maximum achievable throughput, make a match for its corresponding RAU and user, and drop all links related with them. This operation can be mathematically represented as a multiplication of the elements in the same row and column of the selected entry by a binary variable defined as:

$$
\alpha_{k, m}= \begin{cases}1 & \text { if row } k \text { is assigned to column } m \\ 0 & \text { otherwise }\end{cases}
$$

Following this procedure the final match and total sum throughput are $\{(1, b)(2, a)(3, c)\},\{18\}$, respectively. Nevertheless, among all possible matches $\left({ }^{K} \mathbf{P}_{M}\right)$, the optimum solution is given by $\{(1, b)(2, c)(3, a)\}$ that in this context is Pareto optimal ${ }^{2}$ with maximum sum throughput $\{21\}$. The user selection in this scenario is a class of weighted matching problem called linear sum assignment (LSAP) that can be defined as follows [6]:

Given a $K \times M$ matrix $C=\left(c_{k, m}\right)$, the problem is to match each row to a different column in such a way that the sum of the corresponding entries is maximized.

$$
\begin{array}{lll}
\text { maximize } & \sum_{k=1}^{K} \sum_{m=1}^{M} \alpha_{k, m} c_{k, m} & \\
\text { subject to } & \sum_{m=1}^{M} \alpha_{k, m}=1 & (k=1, \ldots, K) \\
& \sum_{k=1}^{K} \alpha_{k, m}=1 & (m=1, \ldots, M)
\end{array}
$$

where $\alpha_{k, m}$ is the weight of the element $c_{k, m}$ defined by (4).

In literature of combinatorial optimization (e.g., [6], [8]) the optimum solution of (5) can be found by several approaches (primal-simplex algorithms, primal-dual algorithms, shortest path algorithms, etc.) We propose low-complexity algorithms that can find a solution to (5) close to the Pareto optimal.

\section{THE PROPOSED ALGORITHMS}

The process of scheduling starts when the users report their channel gains to the $\mathrm{CU}$. We evaluate the achievable

${ }^{2} \mathrm{~A}$ Pareto optimal allocation is one such that, there does not exist another feasible allocation where at least one user gets a better resource assignment (RAU, carrier, etc), and all others get at least the same resources. This optimal allocation cannot be improved on without hurting at least one user [7]. 
throughput $R_{k, m}$ of user $k$ and we assume that RAU $m$ transmits the desired signal and all other RAUs are sources of interference. The terms $R_{k, m}, \forall k \in V_{2}, \forall m \in V_{1}$ are represented in the $C \in \mathbb{R}^{K \times M}$ matrix. Our goal is to assign one user to each RAU meeting the constraints in (6) and (7).

\section{A. First Phase: user-RAU matching}

The entries of the matrix $C$ are defined by the wireless channels. For a given channel realization and user deployment, the information in matrix $C$ can reflect two different conditions that may happen simultaneously. In the first condition, the matrix $C$ has dominant elements in $V_{2}$ such that every user $i$ has $c_{i, m} \gg c_{i, n}, \forall m, n \in V_{1}, n \neq m$. In this case the user selection can be performed in a greedy fashion due to the fact that each user has only one link with high achievable throughput value. In the second condition, let us assume that for some elements in $V_{2}$ there are several links with large achievable throughput such that $c_{i, m} \approx c_{i, n}, \forall m, n \in$ $V_{1}^{\prime}, V_{1}^{\prime} \subseteq V_{1}, n \neq m$, i.e. that more than one element in $V_{1}$ is feasible to serve user $i$.

In the greedy selection for each step of the scheduling process, the user with maximum achievable throughput $k$ attached to RAU $m$ is selected and all links related to that specific user and RAU are dropped as described in Algorithm 1a. This means that when a match between user $k$ and RAU $m$ is found, all possible matches (with good and poor achievable throughput) for $k$ and $m$ are lost. Therefore, by being greedy, in each iteration we can lose resources that would be assigned in the future iterations. Consequently, we propose a minimumthroughput loss algorithm where each time a match is found, it minimizes the losses of such selection by finding the match that will provide the maximum amount of available throughput for the next iteration.

Let us define $c^{(T)}$ as the total available throughput in the cell computed as:

$$
c^{(T)}=\sum_{k} \sum_{m} c_{k, m}
$$

where $c_{k, m}$ is the element in row $k$ and column $m$ in $C$. If a link $(i, j)$ is selected, the amount of total available throughput that will be discarded by this selection, is evaluated as:

$$
c_{i, j}^{(D)}=\sum_{k} c_{k, j}+\sum_{m \neq j} c_{i, m}
$$

The remaining throughput in the system when link $(i, j)$ is selected, is given by $c_{i, j}^{(A)}=c^{(T)}-c_{i, j}^{(D)}$. For the minimumthroughput-loss approach described in Algorithm $1 \mathrm{~b}$, the matching is performed over the matrix $\tilde{C}$ and its elements are evaluated by applying the following transformation:

$$
\tilde{c}_{k, m}=c_{k, m}\left(\frac{c_{k, m}^{(A)}}{c_{k, m}^{(D)}}\right)
$$

where the quotient that multiplies the original term $c_{k, m}$ is the relative gain of the available throughput over the throughput loss for the $(k, m)$ match. The problem (5) is solved by using the outputs of Algorithms 1a and 1b. After applying both
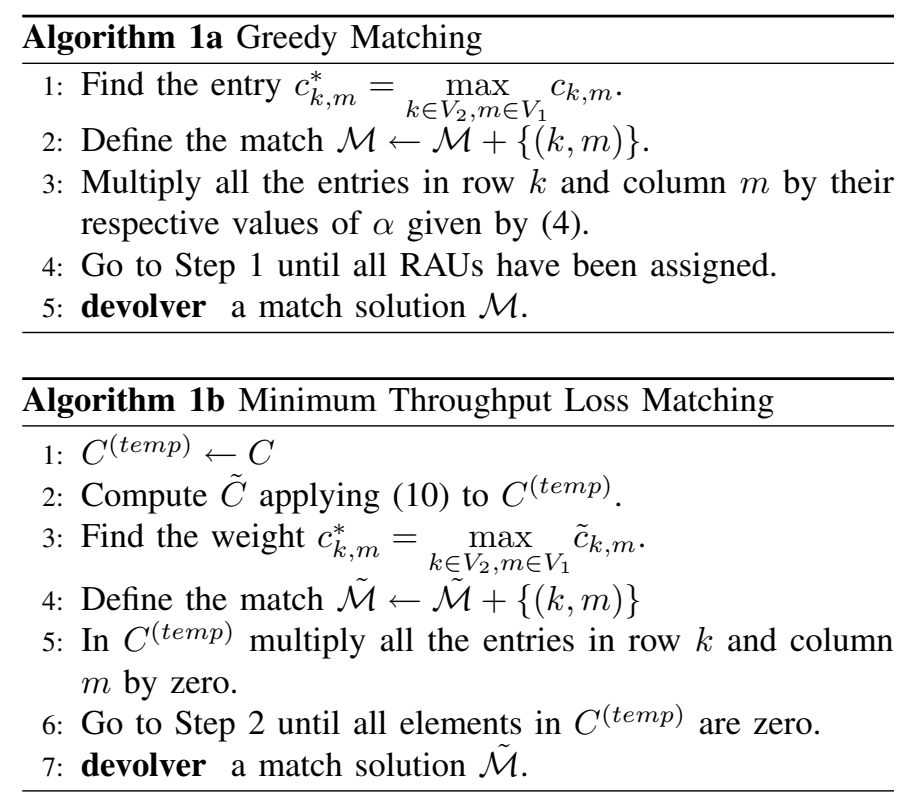

approaches, there are two possible solutions $\mathcal{M}$ and $\tilde{\mathcal{M}}$. The final selected match is the one with maximum sum throughput, and the set of potential users that can be scheduled is given by $\mathcal{K}=\left\{k_{1}, \ldots, k_{|\mathcal{K}|}\right\}$ with $|\mathcal{K}| \leq M$.

\section{B. Second Phase: scheduling and throughput maximization}

For the set of selected users $\mathcal{K}$ there exist $L=\sum_{m=1}^{M}{ }^{\mid \mathcal{K}} \mid \mathbf{C}_{m}$ subsets $S_{(\mathcal{K})}^{(l)}, l \in\{1, \ldots, L\}$ that represent all possible ways that elements in $\mathcal{K}$ can be scheduled with the constraint that each user can be served only by its previously assigned RAU. It is possible that the throughput is maximized by a subset $S_{(\mathcal{K})}^{\left(l^{\text {opt }}\right)}$ such that:

$$
\left(l^{\text {opt }}\right)=\arg \max _{l \in\{1, \ldots, L\}}\left\{R\left(S_{(\mathcal{K})}^{(l)}\right)\right\}
$$

where the term $R\left(S_{(\mathcal{K})}^{(l)}\right)$ is the sum throughput achieved by subset $S_{(\mathcal{K})}^{(l)}$ and is given by:

$$
R\left(S_{(\mathcal{K})}^{(l)}\right)=\sum_{i \in S_{(\mathcal{K})}^{(l)}} \log \left(1+\gamma_{k_{i}, m_{k_{i}}}\right)
$$

where $k_{i}$ is the $i$ th user in subset $S_{(\mathcal{K})}^{(l)}$ and $m_{k_{i}}$ is its associated RAU. If the number of RAUs is large, the evaluation of (12) for all $L$ subsets may be unfeasible. In order to estimate $S_{(\mathcal{K})}^{\left(l^{o p t}\right)}$ without computing $L$ possible combinations an iterative process drops users based on the minimum throughput contribution criterion. This algorithm finds the user for which the downlink transmission generates more interference to the other users compare with his own achievable throughput. This is done evaluating the individual throughput by (3) and calculating the total sum throughput by (12). Then, it is considered that the user with minimum value for (3) is dropped and (3) and (12) are evaluated for all other users. At the end, the user with minimum throughput contribution is dropped 
if the total sum throughput is larger when such user is not scheduled. This process is summarized in Algorithm 2.

For the user-RAU matching, the time complexity is $O\left(K M^{2}\right)$ considering that each matching implies a process of complexity $O(K M)$ and this process is repeated $M$ times. Nevertheless, for DAS where the number of distributed antennas is small and $K \geq M$, the complexity of solving the matching problem is limited. Even if the time complexity of our user assignment algorithm is $O\left(K M^{2}\right)$, its computational complexity is low compared to the complexity of the algorithms that find the optimum solution for (5). The proposed maximization of (12) implies a linear search that is given by the cardinality of the set of matched users $O(|\mathcal{K}|)$.

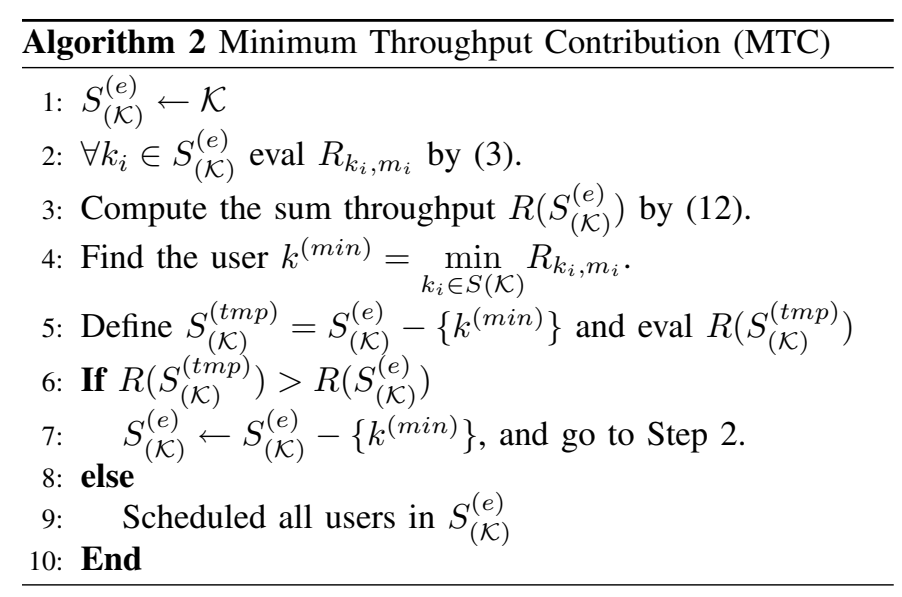

\section{Performance Evaluation}

The deployment of the distributed antennas consist of one RAU at the center of the cell and three distributed RAUs uniformly deployed at a distance from the cell center of $\frac{2}{3}$ the cell radius. The simulation parameters are listed in Table I. The Gini index $\left(F_{G}\right)$ is used to estimate the fairness in the distribution of the throughput among selected users. The $F_{G}$ values lie in the interval $[0,1)$, having $F_{G}=0$ as the maximum level of fairness. For $K$ active users in the system, $F_{G}$ over all channel realizations is given by [9]:

$$
F_{G}=\frac{1}{2 K \sum_{k=1}^{K} \bar{R}_{k}}\left(\sum_{k=1}^{K} \sum_{i=1}^{K}\left|\bar{R}_{k}-\bar{R}_{i}\right|\right)
$$

where $\bar{R}_{k}$ is the average throughput achieved by the $k$ th user.

The tested algorithms were analyzed in terms of different performance metrics: the average sum throughput, the cardinality of the final set of scheduled users, and the fairness of the average achieved throughput. Our algorithm to match user-RAU (Alg.1a-b) defined in the first phase was compared to the generalized Jonker-Volgenant algorithm (JVA) for nonsquare dense matrices [8] (which finds the optimum solution of (5)), and to the dominant user grouping (DUG) algorithm for throughput-maximization [5].

The output of the algorithms to make the user-RAU matching was combined in the second phase with three approaches that establish the final subset of scheduled users: $1 . S_{(\mathcal{K})}^{(e)}$ is
TABLE I: Simulation Parameters

\begin{tabular}{cc}
\hline Parameters & Values \\
\hline Cell radius & $900 \mathrm{~m}$ \\
Carrier frequency & $2.5 \mathrm{GHz}$ \\
Channel bandwidth & $20 \mathrm{MHz}$ \\
RAU transmit power & $43 \mathrm{dBm}$ \\
Thermal noise power density & $-174 \mathrm{dBm}$ \\
UE noise figure & $7 \mathrm{~dB}$ \\
Path loss model & UMi-LoS [10] \\
Shadow fading standard deviation & $\sigma_{s}=3$ \\
User deployment & uniform \\
\hline
\end{tabular}

optimum among all $L$ subsets and maximizes (12), 2. $S_{(\mathcal{K})}^{(e)}$ is found by MTC, 3. $S_{(\mathcal{K})}^{(e)}=\mathcal{K}$, i.e. all matched users from first phase are scheduled.

Fig. 2 shows the average sum throughput for different values of $K$ and $M=4$ achieved by Algorithms Alg.1a-b and JVA. The evaluations show that our algorithm (Alg.1a-b) in all performed simulations achieved the same average performance of JVA for the user-RAU matching in the first phase. Fig. 2 also shows the three approaches to define $S_{(\mathcal{K})}^{(e)}$ and the gap in terms of throughput when the subset of scheduled users is found by MTC and when all matched users in the first phase are scheduled. For $K=10$ the gap in performance between the optimum set compared to $S_{(\mathcal{K})}^{(e)}=\mathcal{K}$ is up to $15 \%$. Nevertheless, the throughput loss incurred by not maximizing (12) is compensated by scheduling more user which is a gain in terms of fairness.

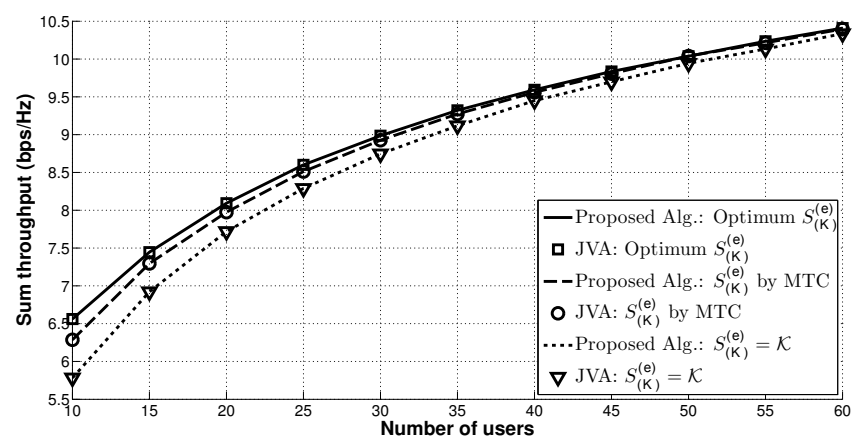

Fig. 2: Sum throughput for $M=4$, different values of $K$.

Fig. 3 shows the expected value of the cardinality of the subset of scheduled users over $M, \mathbb{E}\left[\left|S_{(\mathcal{K})}^{(e)}\right| / M\right]$. For lower values of $K$ the percentage of scheduled users in the optimum subset $S_{(\mathcal{K})}^{(e)}$ can be up to $30 \%$ below the number of users scheduled when $S_{(\mathcal{K})}^{(e)}=\mathcal{K}$. If the subset $S_{(\mathcal{K})}^{(e)}$ is found by MTC, we improved in $15 \%$ the number of scheduled users compare to the optimum subset and the loss in sum throughput is only of 5\%. Table II shows the comparison in terms of sum throughput for $S_{(\mathcal{K})}^{(e)}=\mathcal{K}$ between Alg.1a-b and DUG for low values of $K$. The performance gap between Alg.1a-b and DUG in terms of throughput is less than 3\% but for Alg.1a-b the percentage of scheduled users is $100 \%$. These differences 


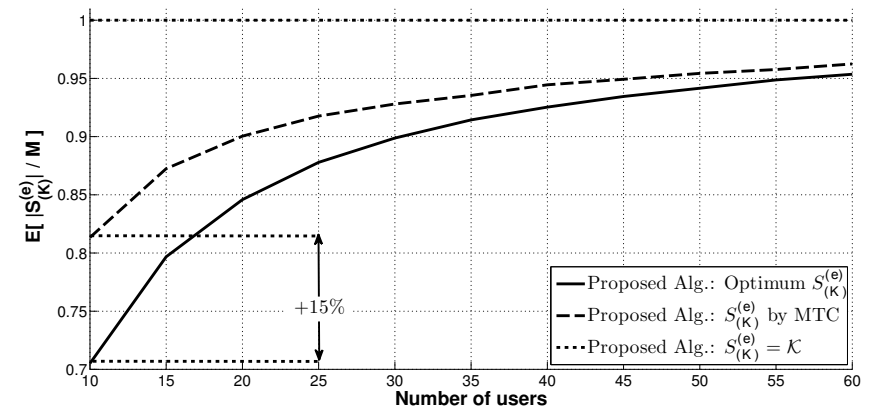

Fig. 3: Percentage of user assignment $\mathbb{E}\left[\left|S_{(\mathcal{K})}^{(e)}\right| / M\right]$ for several values of $K$ and different sets $S_{(\mathcal{K})}^{(e)}$.

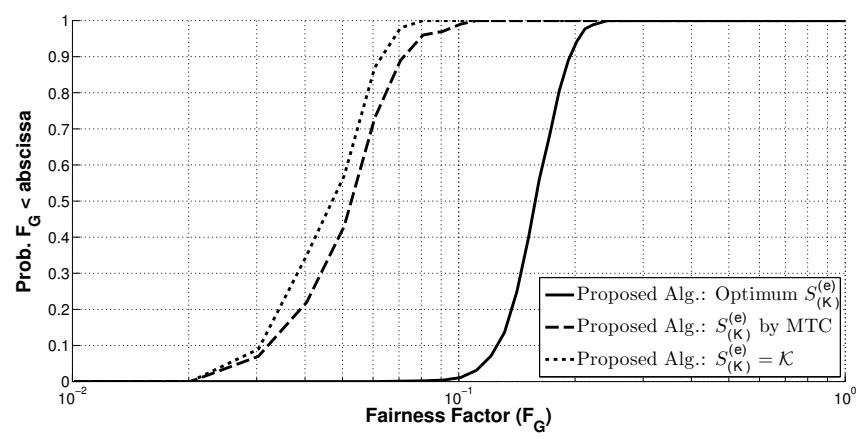

Fig. 4: $\mathrm{CDF}$ of the Fairness Gini Index for $K=10$ and $M=4$.

between Alg.1a-b and DUG algorithms become negligible for large values of $K$.

The throughput distribution among users measured by (13) is shown in Fig. 4. The results reflect the contradicting objectives of maximizing (12) and scheduling all users found in the first phase. It can be observed that by defining the subset of scheduled users by MTC, a trade-off between throughput (a gap of 5\% compared with the optimum subset) and fairness is achieved. The CDF of $F_{G}$ when MTC is applied, is close to the $\mathrm{CDF}$ that gives resources to all user found in the first phase by Alg.1a-b. These results are equal when $\mathcal{K}$ is found by JVA in the fist phase and the sum throughput is maximized by MTC. For large values of $K(K \geq 30)$ the gap in terms of throughput between all possible strategies to find $S_{(\mathcal{K})}^{(e)}$ decreases. Fig. 5 shows the CDF of the achieved throughput for $K=30$. The gap in performance between the scheduling done by MTC and the optimum scheduling is negligible. Moreover, by scheduling all users found by Alg.1a-b in the first phase the throughput gap is only $4 \%$ compared to the optimum scheduling with the advantage that $100 \%$ of the matched users are served.

TABLE II: Comparison between Alg.1a-b and DUG for $R\left(S_{(\mathcal{K})}^{(e)}\right)$ and $\mathbb{E}\left[\left|S_{(\mathcal{K})}^{(e)}\right| / M\right]$ for low values of $K$ and $S_{(\mathcal{K})}^{(e)}=\mathcal{K}$.

\begin{tabular}{cccccc}
\hline & $\mathrm{K}$ & 10 & 15 & 20 & 25 \\
\hline \multirow{2}{*}{$R\left(S_{(\mathcal{K})}^{(e)}\right)$} & Alg. 1a-b & 5.78 & 6.92 & 7.71 & 8.29 \\
& DUG & 5.89 & 6.94 & 7.72 & 8.29 \\
\hline \multirow{2}{*}[|S_{(\mathcal{K})}^{(e)}|/M]{} & Alg. 1a-b & 1 & 1 & 1 & 1 \\
& DUG & 0.94 & 0.98 & 0.99 & 0.99 \\
\hline
\end{tabular}

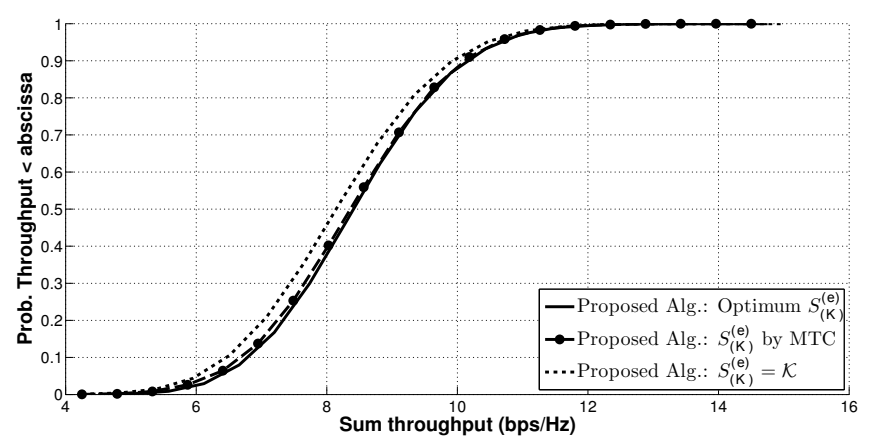

Fig. 5: CDF of the sum throughput for $K=30$ and $M=4$. In this case the set $S_{(\mathcal{K})}^{(e)}=\mathcal{K}$ found by Alg.1a-b can be scheduled with only a $4 \%$ throughput gap compare with the optimum $S_{(\mathcal{K})}^{(e)}$. As $K \rightarrow \infty$ this throughput gap becomes negligible.

\section{CONCLUSION AND FUTURE WORK}

In this paper we proposed an algorithm that operates in two phases, one that combines a greedy matching and a minimumthroughput-loss matching which provides a first set of potential users. The second phase seeks a refine subset of users in order to maximize the total sum throughput. Numerical results show that the performance achieved by our proposed matching algorithm is equal to the optimal solution but with much less complexity. Furthermore, the algorithm allows in the second phase to trade between throughput and fairness depending on the criteria used to set the final subset of scheduled users. Extended work in this area can be done by applying the proposed algorithm to DAS with multiple-antenna per RAU, asymmetric deployment of RAUs, and combining different metrics for user selection such as spatial correlation, fairness, and QoS.

\section{REFERENCES}

[1] H. Hu, Y. Zhang, and J. Luo, Distributed antenna systems: open architecture for future wireless communications. Auerbach Publications, 2007.

[2] E. Driouch and W. Ajib, "A graph theory based scheduling algorithm for MIMO-CDMA systems using zero forcing beamforming," in Computers and Commun. ISCC. IEEE Symposium on, july 2008, pp. $674-679$.

[3] T. Ji, C. Zhou, S. Zhou, and Y. Yao, "Low complex user selection strategies for multi-user MIMO downlink scenario," in Wireless Commun. and Networking Conf. WCNC. IEEE, march 2007, pp. $1532-1537$.

[4] S. Karachontzitis and D. Toumpakaris, "Efficient and low-complexity user selection for the multiuser MISO downlink," in Personal, Indoor and Mobile Radio Commun., IEEE 20th Int. Symposium on, sept. 2009, pp. $3094-3098$.

[5] X. Gao, A. Li, Y. Yan, and H. Kayama, "Dominant users grouping algorithm for multiple RAUs-UEs coordination in DAS system," in Vehicular Technology Conf. Fall, IEEE 72nd, sept. 2010, pp. 1 -5.

[6] R. Burkard, M. Dell'Amico, and S. Martello, Assignment Problems. Siam, 2009.

[7] Z. Han and K. J. R. Liu, Resource Allocation for Wireless Networks: Basics, Techniques, and Applications. Cambridge University Press, 2008.

[8] R. Jonker and A. Volgenant, "A shortest augmenting path algorithm for dense and sparse linear assignment problems," Computing, vol. 38, pp. 325-340, 1987. [Online]. Available: http://dx.doi.org/10.1007/BF02278710

[9] A. W. Marshall and I. Olkin, Inequalites: Theory of Majorization and Its Applications. Academic Press., 1979.

[10] M.2135-1. Guidelines for evaluation of radio interface technologies for IMT-Advanced, ITU-R, 2009. 